\title{
Organotin Dithiohydrazodicarbonamides as Corrosion Inhibitors for Mild Steel-Dimethyl Sulphoxide Containing HCl
}

\author{
R.B. Rastogi*, M.M. Singh, K. Singh, M. Yadav \\ Department of Applied Chemistry, Institute of Technology, Banaras Hindu University, \\ Varanasi, 221005, India
}

Received 14 April 2004; accepted in revised form 02 December 2004

\begin{abstract}
A few 1-aryl-2,5-dithiohydrazodicarbonamides and their triphenyltin and dibutyltin complexes have been studied as corrosion inhibitors for mild steel in dimethyl sulphoxide containing $\mathrm{HCl}$ at $25{ }^{\circ} \mathrm{C}$ using electrochemical polarisation technique. Among dithiohydrazodicarbonamides, ethoxyphenyl derivative exhibited maximum inhibition efficiency (IE) followed by benzyl and phenyl analogues. The same order of IE was observed in their corresponding organotin complexes; however, IE of a complex was found to be significantly higher than that of the constituents ligand. All organotin complexes behaved predominantly as cathodic inhibitors. Triphenyltin complexes proved to be better corrosion inhibitors than their dibutyltin analogues.
\end{abstract}

Keywords: Organotin dithiohydrazodicarbonamides, corrosion inhibitors, mild steelDMSO, $\mathrm{HCl}$.

\section{Introduction}

Dithiohydrazodicarbonamides have been successfully employed as inhibitors for corrosion of copper in aqueous chloride solution [1,2]. These compounds and their molybdenum and tungsten complexes have been reported as excellent

\footnotetext{
* Corresponding author.
} 
extreme pressure lubricant additives [3] as well as corrosion inhibitors for mild steel-1.0 N sulphuric acid system [4]. Organotins are also known for their corrosion inhibiting properties [5-8]. It is an established fact that complexes, in general, exhibit better corrosion inhibiting tendencies as compared to their respective ligands [9-12]. A few organotin complexes of dithiohydrazodicarbonamides thereby are being used here as corrosion inhibitors for mild steel-dimethyl sulphoxide containing $\mathrm{HCl}$.

\section{Experimental}

The kinetics of corrosion inhibition of mild steel in dimethylsulfoxide medium with $0.2 \mathrm{M}$ hydrochloric acid was studied using tetraethylammonium chloride as the supporting electrolyte in the absence and presence of various concentrations of inhibitors at $25{ }^{\circ} \mathrm{C}$ by potentiostatic polarization technique. The results obtained from these experiments have been used to explain the mechanism of corrosion inhibition.

The polarization experiments were carried out in a three-necked double walled pyrex glass assembly. The cell was air-tight, containing an inlet with variable depth for nitrogen gas and an outlet with $\mathrm{CaCl}_{2}$ tube attached to it. The three electrode cell assembly consisted of a working electrode of mild steel $\left(1 \mathrm{~cm}^{2}\right.$ exposed area), a saturated calomel electrode with $\mathrm{KNO}_{3}$ salt bridge as a reference electrode and a platinum counter electrode. The working and reference electrodes were kept very close to each other in order to minimize IR drop. Water, at a constant temperature from a thermostatic water-bath, was circulated through the space between the two walls to maintain the temperature of the cell. For applying different potentials across the working and reference electrodes, use was made of the Wenking model POS 73 potentiostat and steady state current values were recorded from the ammeter on the panel of the potentiostat.

The working electrode specimens for electrochemical experiments were prepared from mild steel sheets having the following percentage composition:

\begin{tabular}{|c|c|c|c|c|c|c|c|c|}
\hline $\mathrm{C}$ & $\mathrm{Mn}$ & $\mathrm{Si}$ & $\mathrm{P}$ & $\mathrm{S}$ & $\mathrm{N}$ & $\mathrm{Cu}$ & $\mathrm{Cr}$ & $\mathrm{Fe}$ \\
\hline 0.12 & 0.11 & 0.02 & 0.02 & 0.02 & 0.02 & 0.01 & 0.01 & reminder \\
\hline
\end{tabular}


The specimens were mechanically polished successively with 1/0, 2/0, 3/0 and 4/0 grades of emery papers. After this, the surface was thoroughly washed with soap, running tap water, distilled water and finally was degreased with acetone. The samples were dried and stored in a vacuum desiccator before immersing in the test solution.

The solvent DMSO (E. Merck) was first treated with alumina and then was distilled three times under reduced pressure according to the reported method [13]. The solutions were prepared by dissolving corresponding volumes of standard hydrochloric acid ( $\mathrm{HCl}$ was standardized titrimetrically) in DMSO using 0.1 M tetraethylammonium chloride as supporting electrolyte.

The polarization studies were performed in dimethylsulfoxide $0.02 \mathrm{M}$ hydrochloric containing $0.1 \mathrm{M}$ tetraethylammonium chloride as supporting electrolyte, with and without the inhibitors. Before starting the polarization experiment, the open circuit potential (OCP) at the metal-electrolyte interface was noted. After determining the OCP, the potential was applied manually in steps of $10 \mathrm{mV}$ in the anodic or cathodic direction and the corresponding steady state current densities were measured. Fresh samples were used for both types of polarization experiments. Triplicate, some times even more, experiments were run on fresh samples in order to check the reproducibility of the results.

Anodic and cathodic polarization experiments were performed in DMSO solutions containing various concentrations of inhibitors. The corrosion current density ( $i_{\text {corr }}$ ) was determined using the Tafel extrapolation method [14]. The corrosion rate was evaluated by using equation, $0.1288 \mathrm{i}_{\text {corr }}(\mathrm{E} / \mathrm{D}$, when $\mathrm{E}$ stands for electrochemical equivalent and $\mathrm{D}$ for density $\mathrm{g} \mathrm{cm}^{-3}$ ).

For the determination of inhibition efficiencies (I.Es.), the following equation was used

$$
\text { \%I.E. }=100 \times\left(\mathrm{i}_{\text {corr }}^{0}-\mathrm{i}_{\text {corr }}\right) / \mathrm{i}_{\text {corr }}^{0}
$$


where $\mathrm{i}_{\text {corr }}^{0}$ is the corrosion current density $\left(\mu \mathrm{A} \mathrm{cm}^{-2}\right)$ in the absence of inhibitor and $\mathrm{i}_{\text {corr }}$ is the corrosion current density $\left(\mu \mathrm{A} \mathrm{cm}^{-2}\right)$ in the presence of inhibitors.

Dithiohydrazodicarbonamides were prepared by the reported method [4]. Organotin complexes were prepared by mixing methanolic solutions of organotin chloride and corresponding ligand in appropriate ratio with continuous stirring. The reaction mixture was then refluxed for 2 hours and the excess of solvent was distilled off under reduced pressure. The residue was washed with benzene and extracted into chloroform and filtered. The crude product obtained after evaporation of chloroform was recrystallized. The compound was dried in vacuo.

\section{Results and Discussion}

The percentage inhibition efficiency (IE) of 20, 40, 60, 80 and $100 \mathrm{ppm}$ of the ligands, 1-phenyl-2,5-dithiohydrazodicarbonamide (PTHCH), 1-benzyl-2,5dithiohydrazodicarbonamide (BzTHCH), 1-(4-ethoxyphenyl)-2,5dithiohydrazodicarbonamide (EtOPTHCH), their triphenyltin and dibutyltin complexes have been evaluated at $25^{\circ} \mathrm{C}$ and the results are collected in Table 1 .

Table 1. Percentage inhibition efficiency (\% IE) values calculated by polarisation technique in the presence of dithiohydrazodicarbonamides and their triphenyltin (IV) and dibutyltin(IV) complexes.

\begin{tabular}{|c|c|c|c|}
\hline Concentration & $\begin{array}{c}\text { Ligand } \\
\text { PTHCH }\end{array}$ & $(\mathrm{Bu})_{2} \operatorname{Sn}(\mathrm{PTHC})_{2}$ & $(\mathrm{Ph})_{3}$ SnPTHC \\
\hline 20 & 58.31 & 62.19 & 69.19 \\
\hline 40 & 64.24 & 69.46 & 76.12 \\
\hline 60 & 68.37 & 73.70 & 79.84 \\
\hline 80 & 70.48 & 76.55 & 82.46 \\
\hline 100 & 72.87 & 79.80 & 85.43 \\
\hline & $\mathrm{BzTHCH}$ & $(\mathrm{Bu})_{2} \mathrm{Sn}(\mathrm{BzTHC})_{2}$ & $(\mathrm{Ph})_{3} \mathrm{SnBzTHC}$ \\
\hline 20 & 62.12 & 67.42 & 74.19 \\
\hline 40 & 70.34 & 74.12 & 82.13 \\
\hline 60 & 73.70 & 77.26 & 85.43 \\
\hline 80 & 76.55 & 79.80 & 88.34 \\
\hline 100 & 79.80 & 82.13 & 90.26 \\
\hline & $\mathrm{EtOPTHCH}$ & $(\mathrm{Bu})_{2} \mathrm{Sn}(\mathrm{EtOPTHC})_{2}$ & $(\mathrm{Ph})_{3} \mathrm{SnEtOPTHC}$ \\
\hline 20 & 68.54 & 73.70 & 78.12 \\
\hline 40 & 76.28 & 81.12 & 86.18 \\
\hline 60 & 79.80 & 84.34 & 89.43 \\
\hline 80 & 82.13 & 87.42 & 92.67 \\
\hline 100 & 85.94 & 89.43 & 95.17 \\
\hline
\end{tabular}


An increase in IE with increase in concentration of the inhibitors is acknowledged in all cases. The structures of the ligands are given below.

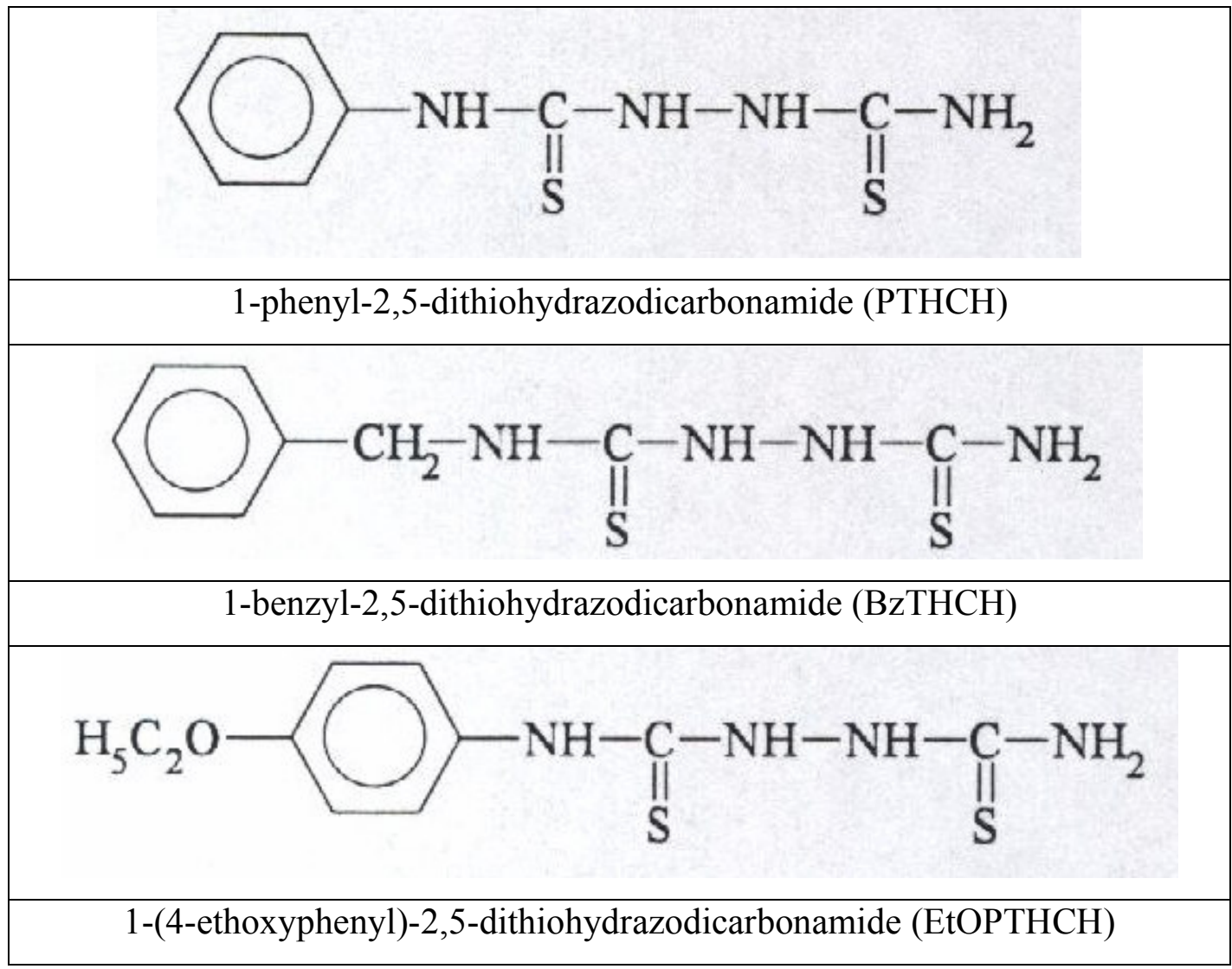

The IE values of these compounds follow the sequence:

$$
\text { EtOPTHCH }>\text { BzTHCH }>\text { PTHCH }
$$

The parent compound PTHCH shows appreciable value of inhibition efficiency due to the presence of phenyl ring, nitrogen and sulphur atoms as centres for adsorption. Introduction of $-\mathrm{OC}_{2} \mathrm{H}_{5}$ group at $p$-position of phenyl ring increases $\pi$ electron density on it, consequently the IE of EtOPTHCH is observed to be maximum. Since benzyl group behaves as an $o, p$-directing alkyl group, the compound BzTHCH shows better IE than the parent compound containing phenyl group. 
It may be inferred from the data collected in Table 1 that there is significant increase in IE values of the complexes as compared to the corresponding ligands; the order of IE of the complexes follows the same pattern as that in case of the respective ligands and lastly triphenyltin complexes appear to be better inhibitors than their dibutyltin analogues.

Thus IEs of the investigated inhibitors may be arranged like

$$
\begin{gathered}
(\mathrm{Ph})_{3} \mathrm{SnEtOPTHCH}>(\mathrm{Ph})_{3} \mathrm{SnBzTHC}>(\mathrm{Ph})_{3} \mathrm{SnPTHC} \\
(\mathrm{Bu})_{2} \mathrm{Sn}(\mathrm{EtOPTHCH})_{2}>(\mathrm{Bu})_{2} \mathrm{Sn}(\mathrm{BzTHC})_{2}>(\mathrm{Bu})_{2} \mathrm{Sn}(\mathrm{PTHC})_{2} \\
(\mathrm{Ph})_{3} \mathrm{SnEtOPTHCH}>(\mathrm{Bu})_{2} \mathrm{Sn}(\mathrm{BzTHC})_{2}>\text { EtOPTHCH }
\end{gathered}
$$

The difference in the behaviour of triphenyltin and dibutyltin complexes may be discussed on the basis of their structures mentioned below:

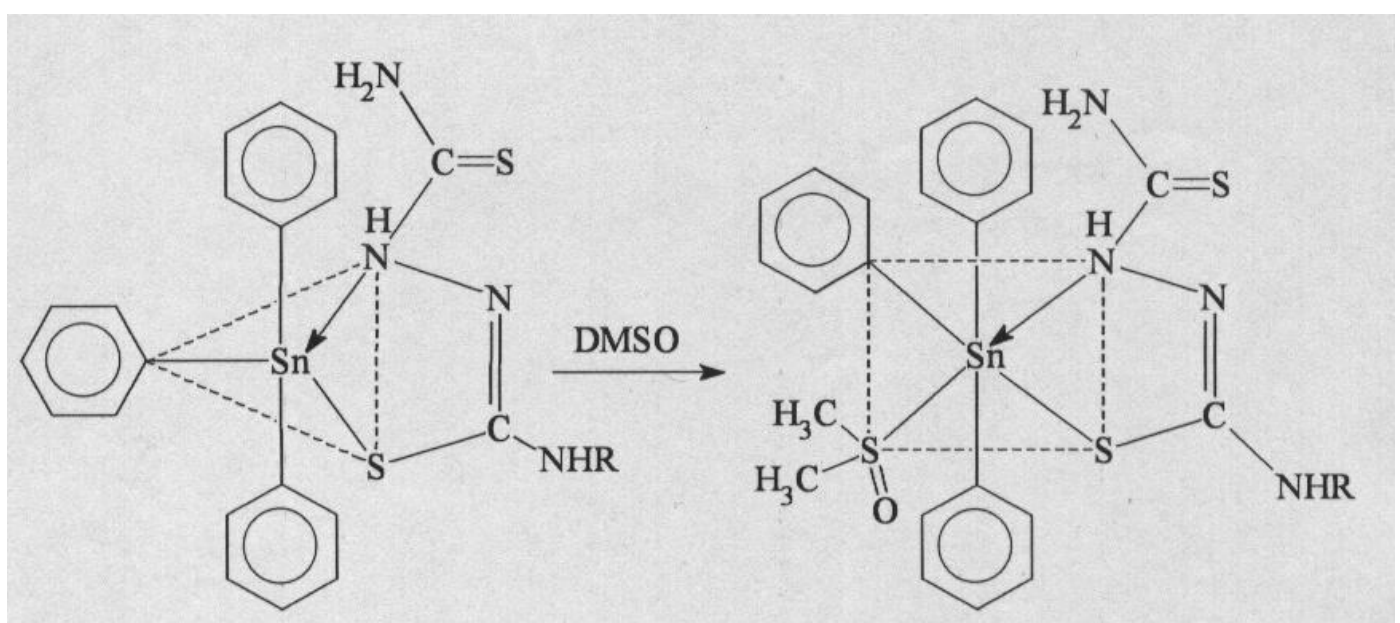

Triphenyltin complex in solid state

Triphenyltin complex in DMSO 


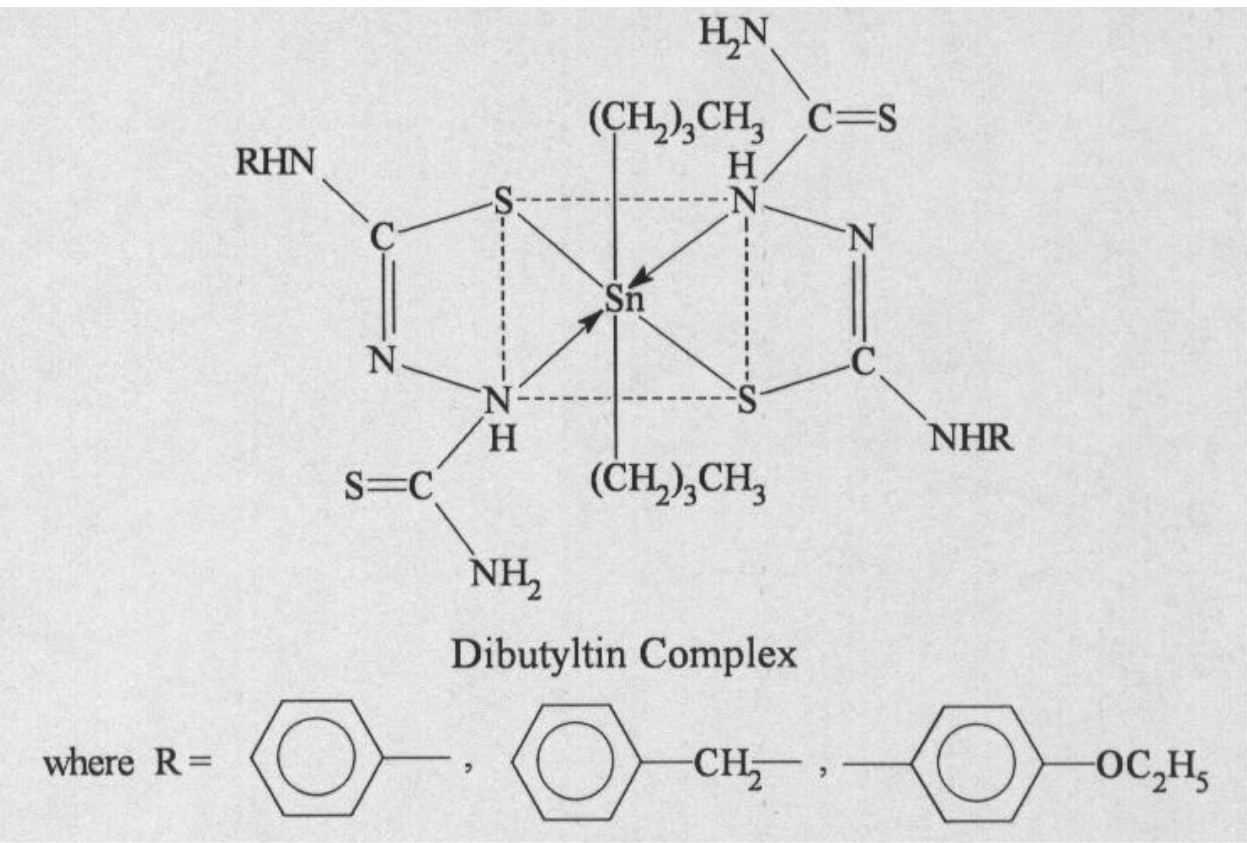

Triphenyltin complex possesses trigonal bipyramidal geometry where the ligand being bulk of occupies two of its equatorial positions and the remaining three positions are occupied by phenyl groups. In the presence of DMSO, a strong coordinating solvent, the geometry of the complex changes from trigonal bipyramidal to octahedral. However, the dibutyltin complexes possess the same octahedral geometry in presence and absence of DMSO. Thus, both the triphenyltin and dibutyltin complexes possess the same octahedral geometry in DMSO but their projected surface area for adsorption on the metal surface may be different. A close look at these structures reveals that in case of dibutyltin complexes which appear symmetrical adsorption would have occurred through one of the ligands only at a time, the other remaining uninvolved. Consequently the expected increase in IE due to the presence of two ligand groups is apparently not observed. On the contrary, triphenyltin complexes with their unsymmetrical structure may get adsorbed on the metal surface through the ligand as well as one of the phenyl rings attached to tin atom increasing thereby the projected surface area and hence the resultant inhibition efficiency. Thus it may be concluded that the observed increase in inhibition efficiency could be correlated well with the increase in projected surface area and the number of active centres involved in adsorption. 
The anodic polarisation behaviour of mild steel in dimethyl sulfoxide system containing different concentrations of the inhibitors has been studied at $25^{\circ} \mathrm{C}$. The curves in Fig. 1 illustrate the anodic polarisation behaviour of mild steel in the presence of $20,40,60,80$ and $100 \mathrm{ppm}$ of 1-phenyl-2,5dithiohydrazodicarbonamide at $25{ }^{\circ} \mathrm{C}$. The nature of the curves does not alter on adding the additive and also on increasing its concentration. The curve shifted appreciably even on the addition of a small amount of the inhibitor (20 ppm). A further increase in concentration of the inhibitor up to $100 \mathrm{ppm}$ though resulted in a further decrease in the current density, but not significantly.

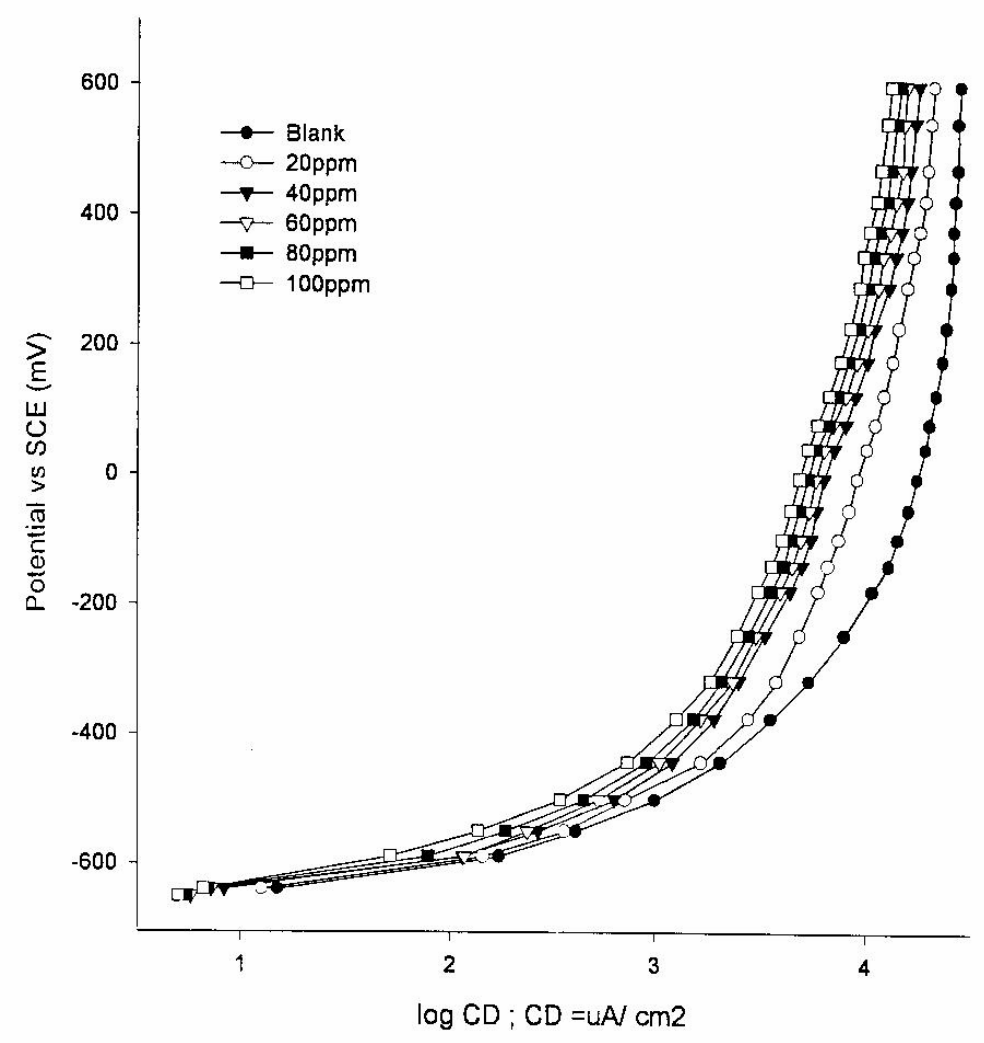

Figure 1. Anodic polarisation behaviour in the absence and presence of different concentrations of 1-phenyl-2,5-dithiohydrazodicarbonamide.

The nature of the anodic polarisation curves and their shifts with the concentration observed for the other inhibitors also exhibit similar trend at all concentrations at $25^{\circ} \mathrm{C}$ and hence, are not being shown. It is realised from these observations that the additive molecules retard the corrosion process without 
changing the mechanism of corrosion process in the medium of investigation. The magnitude of the shift in the current density is a function of the concentrations of the inhibitors, indicating that the inhibitive property of these compounds is concentration dependent.

Fig. 2 illustrates the potentiostatic anodic polarisation curves for all the different dithiohydrazodicarbonamides at a maximum concentration of $100 \mathrm{ppm}$ at $25{ }^{\circ} \mathrm{C}$. The shift towards lower current density was observed to be maximum for ethoxy derivative indicating its maximum effectiveness with respect to its interference with anodic reaction. The observed shift in anodic polarisation curves due to each of the inhibitor follows the same trend as that followed by the inhibition efficiencies.

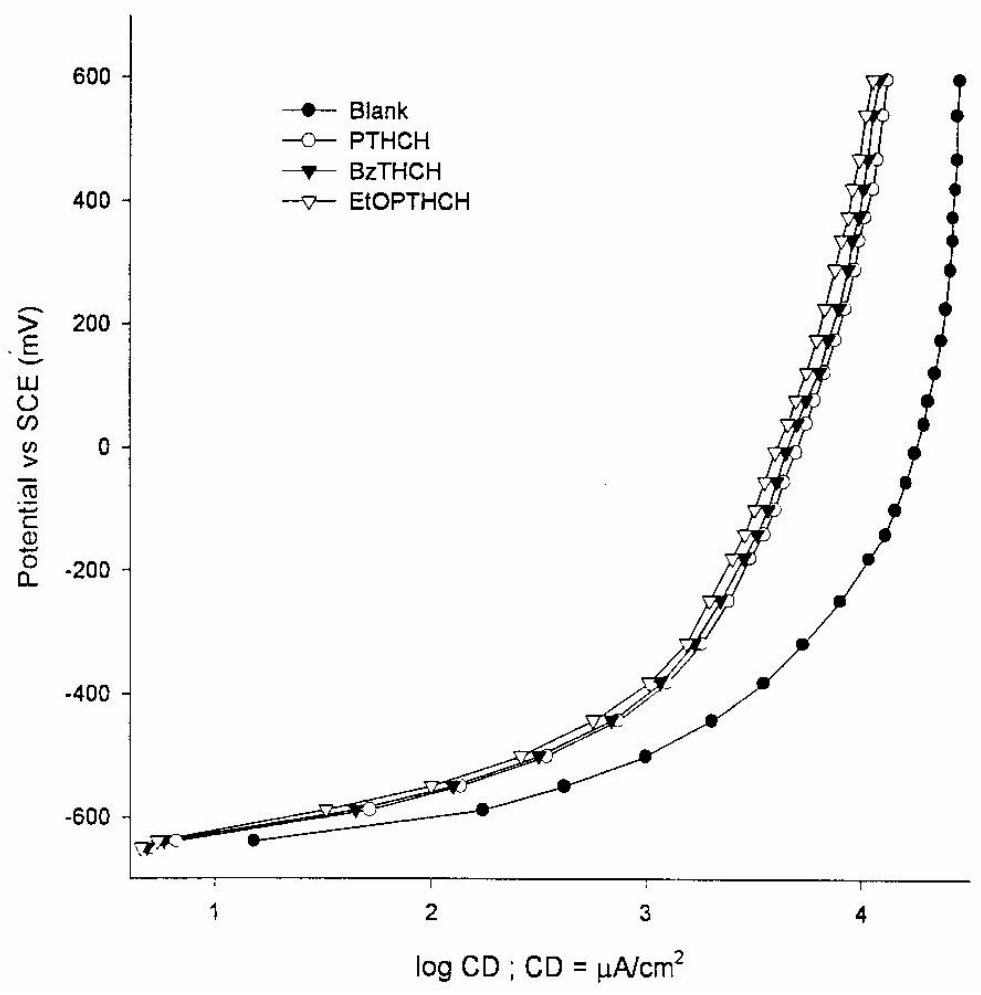

Figure 2. Anodic polarisation beahaviour in the absence and presence of $100 \mathrm{ppm}$ of different derivatives of ligand 1-phenyl-2,5-dithiohydrazodicarbonamide.

The curves in Fig. 3 illustrate the anodic polarisation behaviour of mild steel in the presence of $20,40,60,80$ and 100 ppm of triphenyltin complex of 1-phenyl2,5-dithiohydrazodicarbonamide at $25^{\circ} \mathrm{C}$. The nature of the curves does not alter on adding these additives and also on increasing their concentrations. The shift in 
anodic polarisation curves for the same concentration of different inhibitors occurs in the same sequence for the complexes as for their respective ligands. However, when the ligand was replaced by the corresponding triphenyltin complex, larger shift in polarisation curve was observed, indicating enhanced inhibition efficiency of the complexes.

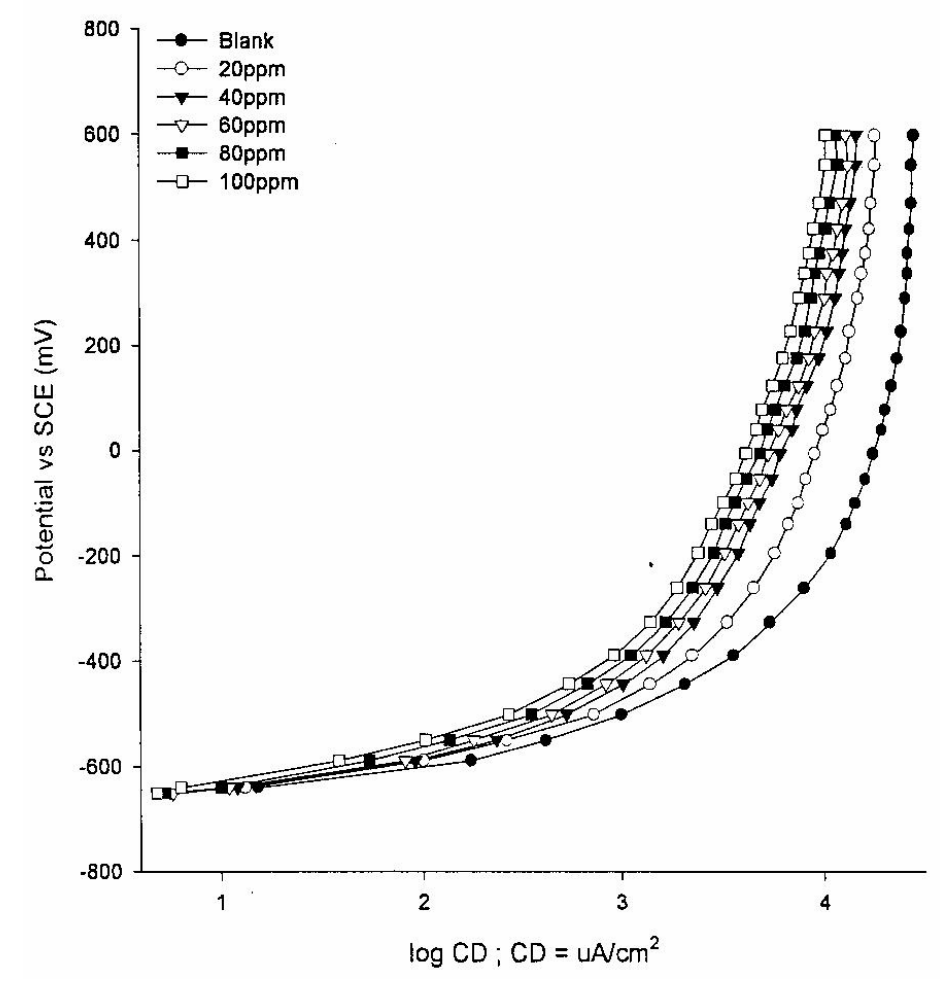

Figure 3. Anodic polarisation behaviour in the absence and presence of different concentrations of $(\mathrm{Ph})_{3} \mathrm{SnPTHC}$. 


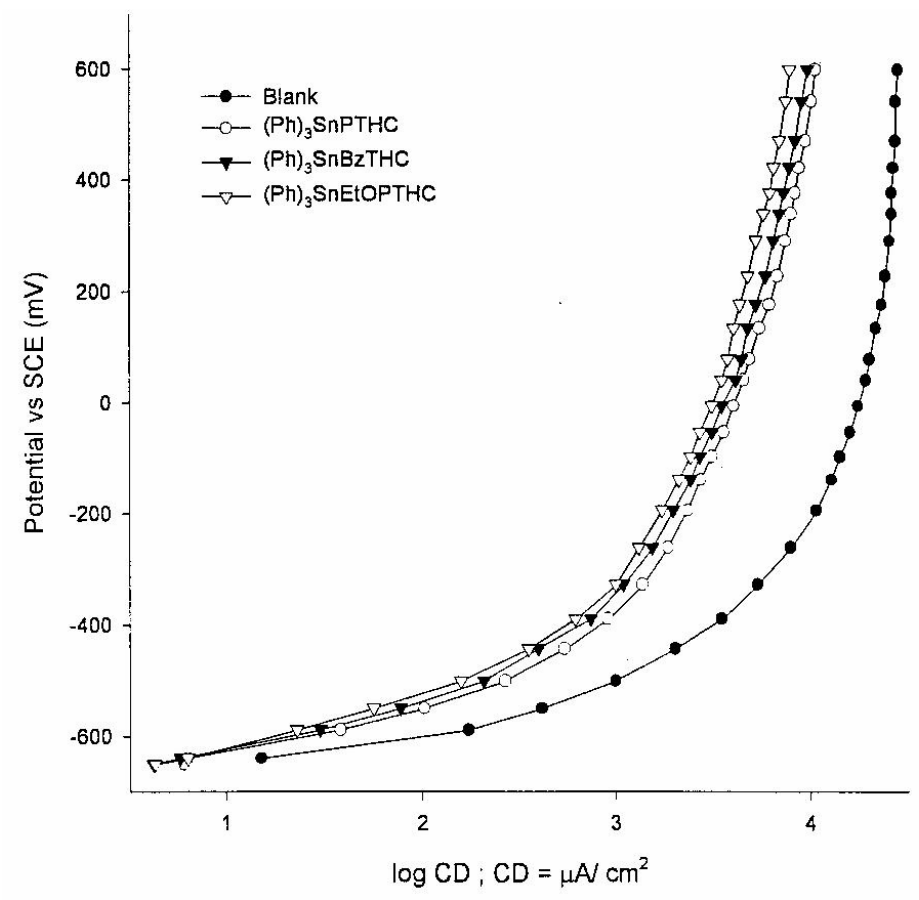

Figure 4. Anodic polarisation behaviour in the absence and presence of $100 \mathrm{ppm}$ of triphenyltin dithiohydrazodicarbonamides.

Fig. 4 illustrates the anodic polarisation behaviour of mild steel in DMSO in the absence and presence of $100 \mathrm{ppm}$ of triphenyltin dithiohydrazodicarbonamides. Although the nature of curves being similar for different compounds, the magnitude of decrease in current density followed the same order as that in case of the corresponding ligands.

Fig. 5 shows the anodic polarisation behaviour of mild steel in DMSO in absence and presence of 20 and $100 \mathrm{ppm}$ of 1-(4-ethoxyphenyl)-2,5dithiohydrazodicarbonamide and its triphenyltin and dibutyltin complexes at 25 ${ }^{\circ} \mathrm{C}$. The maximum decrease in the current density is found for triphenyltin complex followed by dibutyltin complex and the ligand. The nature of the curves is almost similar which indicates that the mechanism of the anodic reaction does not change as a result of replacing one inhibitor by the other. The compound $(\mathrm{Ph})_{3} \mathrm{SnEtOPTHC}$ having more active centres, i.e., four phenyl, one central tin atom and greater $\pi$ electron density in phenyl rings is believed to be adsorbed very strongly on the metal surface. The compound $(\mathrm{Bu})_{2} \mathrm{Sn}(\mathrm{EtOPTHC})_{2}$ has more electron density on the central tin atom as compared to triphenyltin derivative, 
inspite of that triphenyltin complex shows better inhibition efficiency as compared to dibutyltin complex.

The cathodic polarisation curves of mild steel in the presence of different concentrations of 1-phenyl)-2,5-dithiohydrazodicarbonamide at $25{ }^{\circ} \mathrm{C}$ is shown in Fig. 6. It is evident from Fig. 6 that on increasing the concentration of additives the curves shifted towards lower current density region. The observed trend for other additives viz. 1-benzyl-2,5-dithiohydrazodicarbonamide and 1-(4ethoxyphenyl)-2,5-dithiohydrazodicarbonamide is similar at all concentrations at $25^{\circ} \mathrm{C}$. Further at fixed concentration of $100 \mathrm{ppm}$ for these additives as shown in Fig. 7, the relative downward shift is in accordance with their inhibition efficiencies. This indicates that all additives are able to prevent the cathodic process along with the anodic process occurring simultaneously.

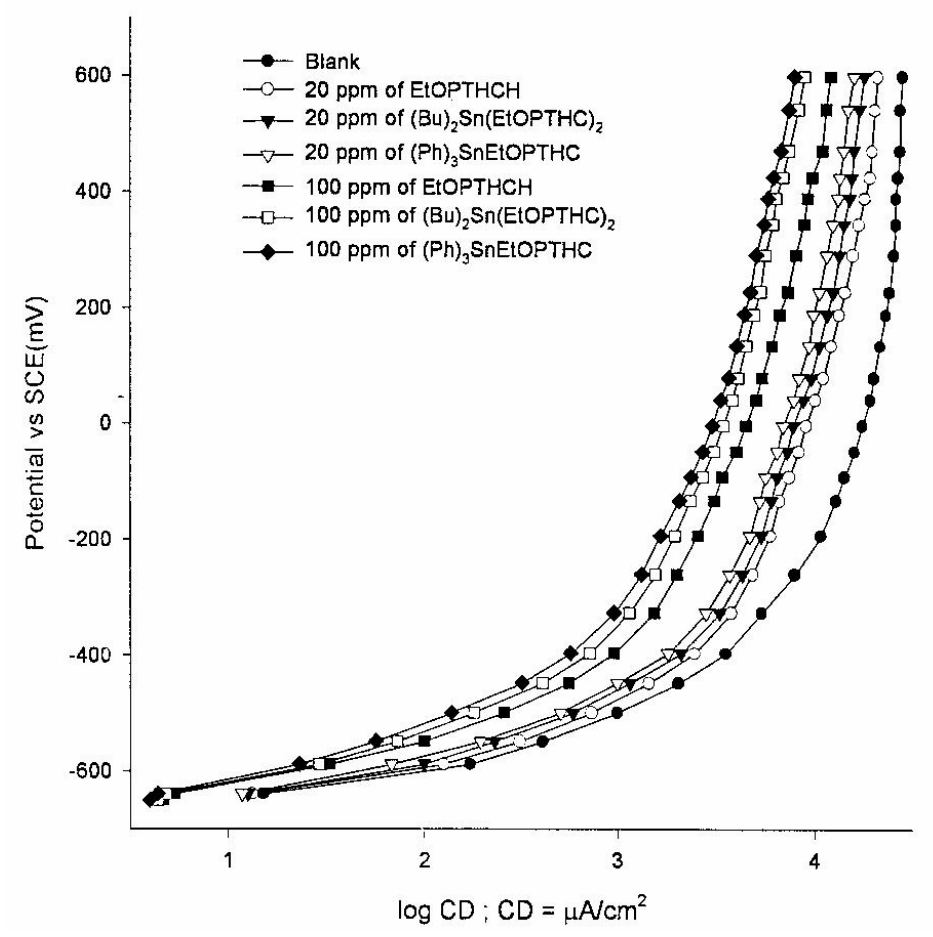

Figure 5. Anodic polarisation behaviour in the absence and presence of 20 and $100 \mathrm{ppm}$ of EtOPTHCH and its organotin complexes. 


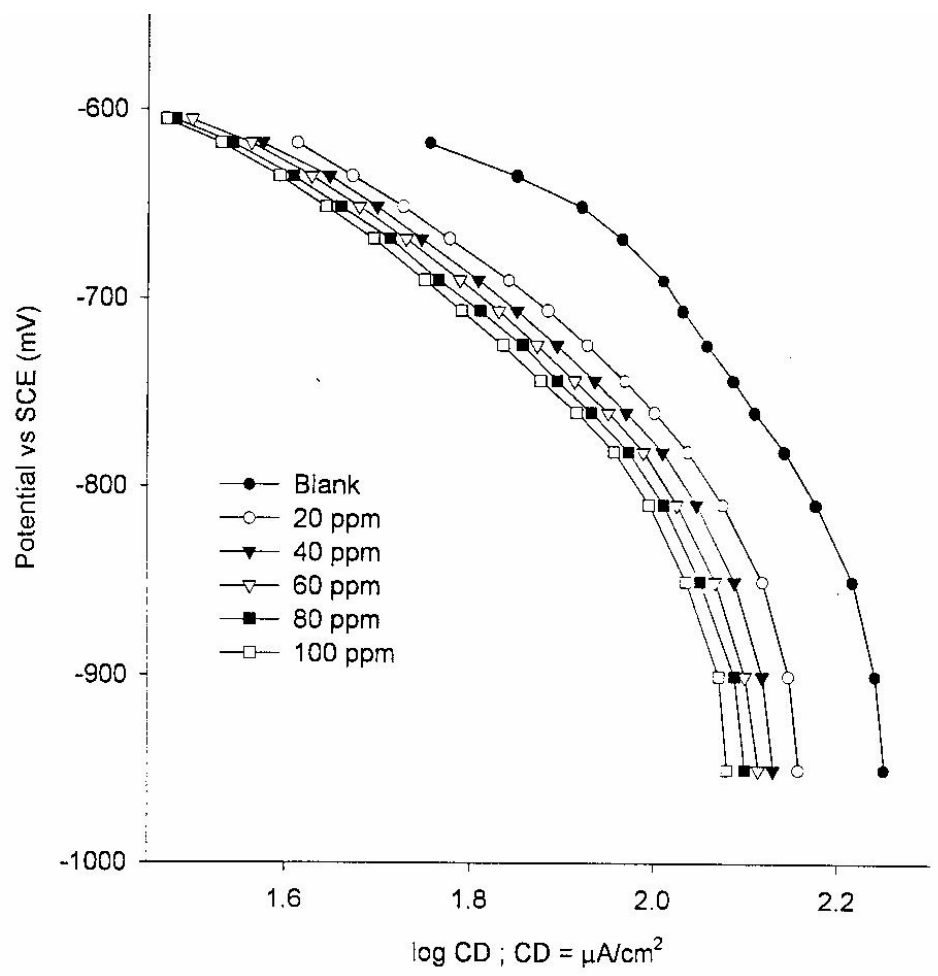

Figure 6. Cathodic polarisation behaviour in the absence and presence of different concentrations of 1-phenyl-2,5-dithiohydrazodicarbonamide.

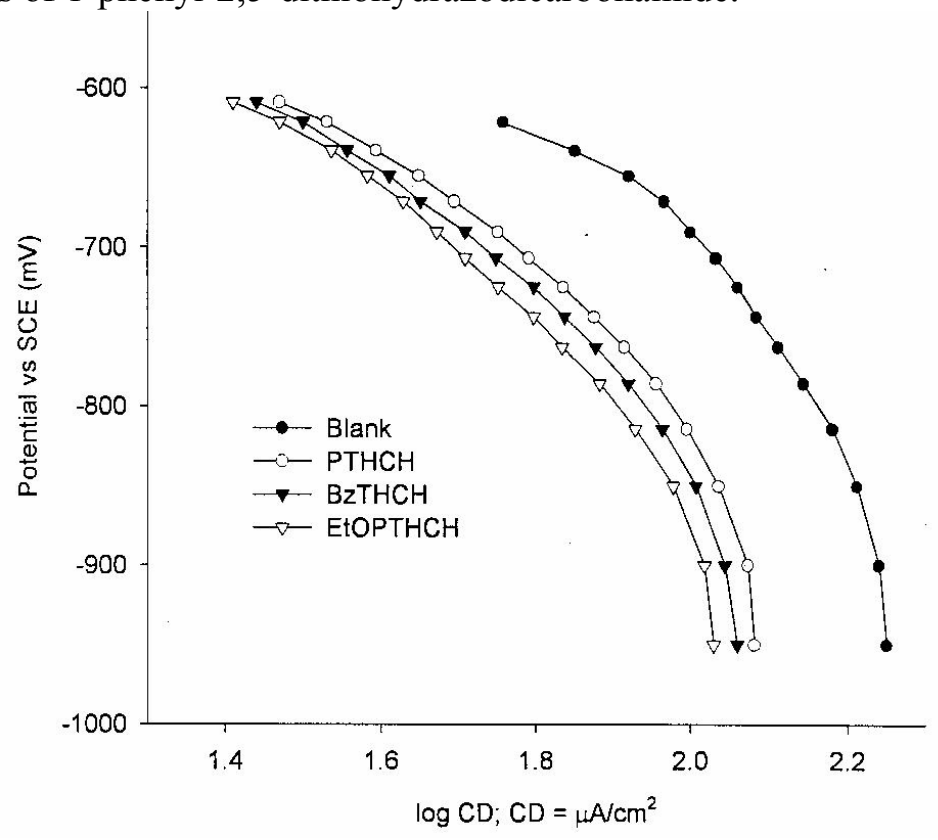

Figure 7. Cathodic polarisation behaviour in the absence and presence of $100 \mathrm{ppm}$ of different dithiohydrazodicarbonamides.

In Fig. 8 it is illustrated the cathodic polarisation behaviour of mild steel in the presence of different concentrations of 1-phenyl-2,5-dithiohydrazodicarbonamide 
complex of triphenyltin at $25^{\circ} \mathrm{C}$. It is evident from Fig. 8 that the extent of the shift of the curves towards lower current density is dependent on the concentrations of the additives and also that the shift is larger as compared to the corresponding ligands. The observed trend for all the triphenyltin complexes at all concentrations at $25^{\circ} \mathrm{C}$ is similar. Further, at fixed concentration of $100 \mathrm{ppm}$ for different triphenyltin complexes the relative downward shifts are in accordance with their inhibition efficiencies. Fig. 9 shows the cathodic polarisation behaviour of mild steel in the presence of triphenyltin dithiohydrazodicarbonamides. It is noted from the figure that the nature of curves remains unaffected with different compounds but the magnitude of shift in current density is directly related with nature of the constituent ligand. Fig. 10 shows the cathodic polarisation behaviour of mild steel in presence of 20 and 100 ppm of $(\mathrm{Ph})_{3} \mathrm{SnEtOPTHC},(\mathrm{Bu})_{2} \mathrm{Sn}(\mathrm{EtOPTHC})_{2}$, and the ligand EtOPTHCH at $25^{\circ} \mathrm{C}$. It is observed from the figure that the shift in the curves follows the same trend as that in the case of anodic polarisation curves. Further, the extent of shifts was found to be much pronounced in the case of cathodic polarisation curves of organotin complexes as compared to those of anodic polarisation curves. This shows that organotin complexes behaved predominantly as cathodic inhibitors.

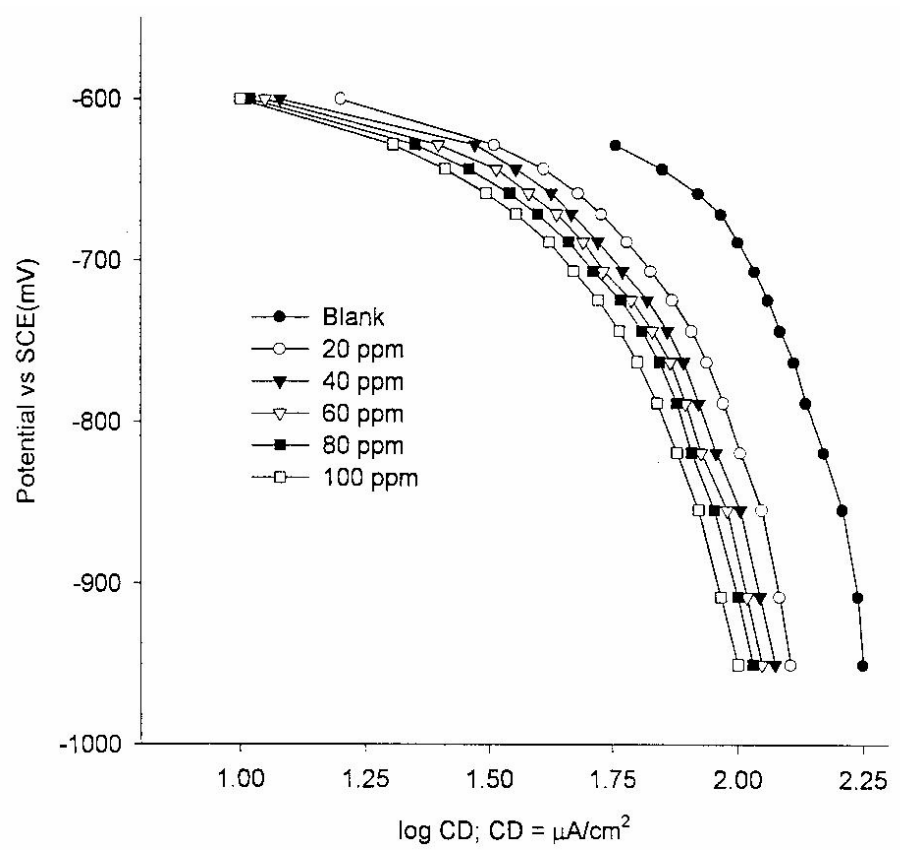

Figure 8. Cathodic polarisation behaviour in the absence and presence of different concentrations of $(\mathrm{Ph})_{3} \mathrm{SnPTHC}$. 


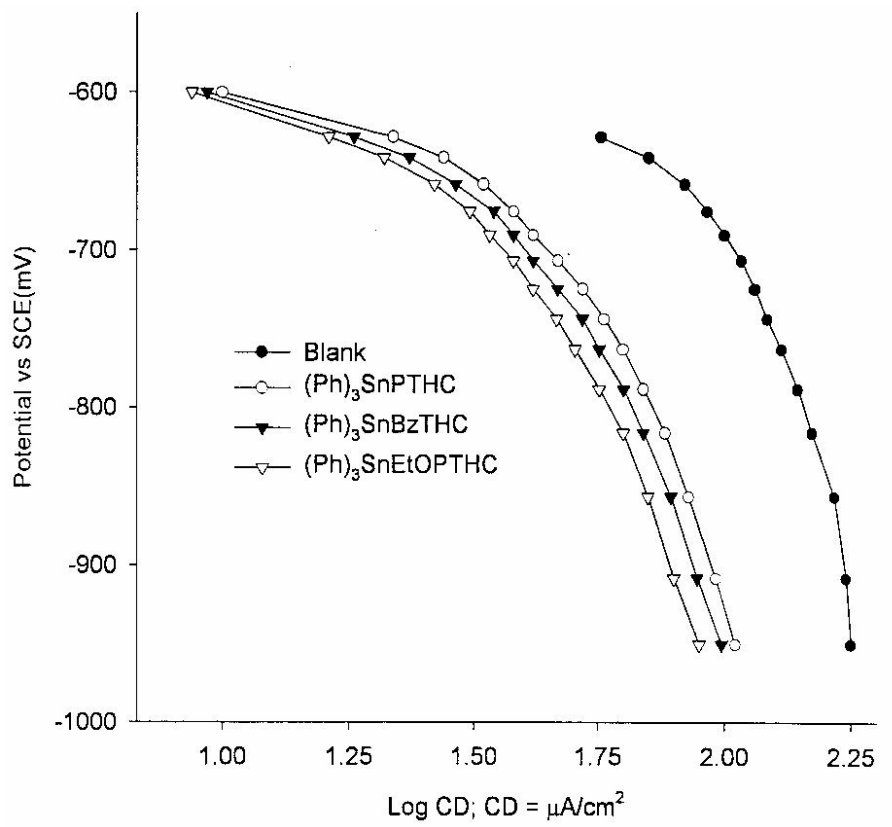

Figure 9. Cathodic polarisation behaviour in the absence and presence of $100 \mathrm{ppm}$ of triphenyltin dithiohydrazodicarbonamides.

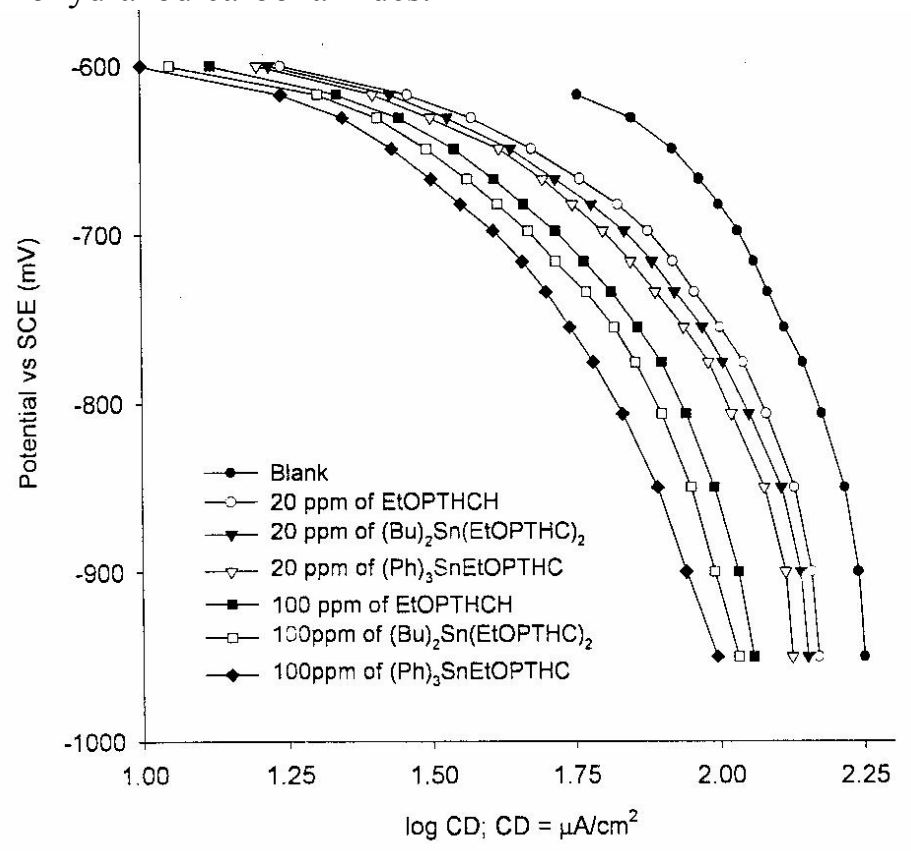

Figure 10. Cathodic polarisation behaviour in the absence and presence of 20 and 100 ppm of EtOPTHCH, Bu $\mathrm{Bu}_{2} \mathrm{Sn}(\mathrm{EtOPTHC})_{2}$ and $(\mathrm{Ph})_{3} \mathrm{SnPTHC}$.

All the investigated inhibitors give a straight line, for $\log (\theta /(1-\theta))$ vs. $\log \mathrm{C}$ plots at $25{ }^{\circ} \mathrm{C}$ as shown in Fig. 11. But these are not ideal Langmuir adsorption isotherm plots as the gradients are never unity. The deviation from unity may be due to mutual attractive or repulsive interactions between adsorbed molecules on 
the metal surface. Further, all the investigated inhibitors show a linear relationship when the values of $\theta$ are plotted against $\log C$, indicating the validity of Temkin's isotherm, Fig. 12 [15].
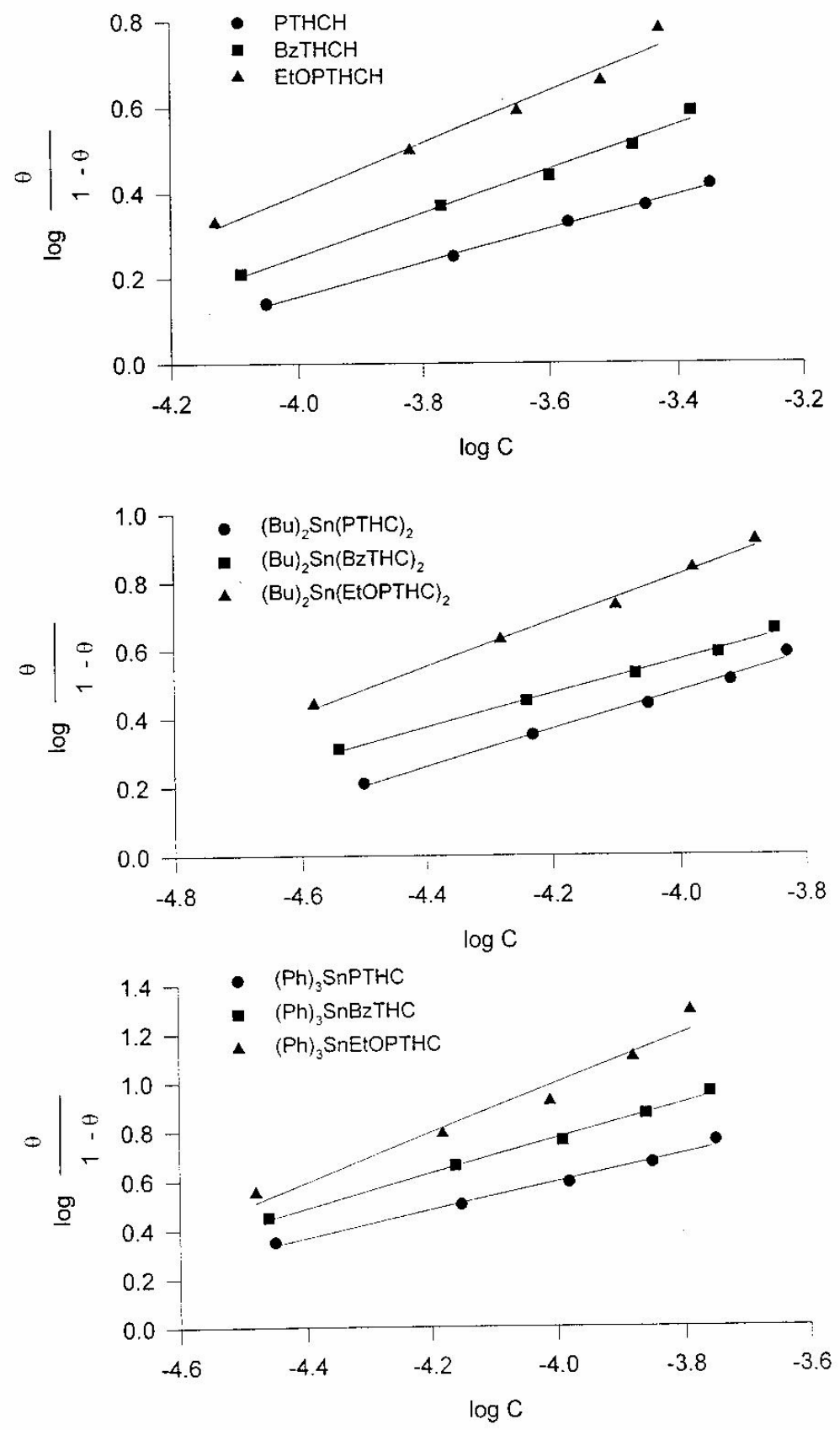

Figure 11. Langmuir plots for dithiohydrazodicarbonamides and their dibutyltin (IV) and triphenyltin (IV) complexes. 

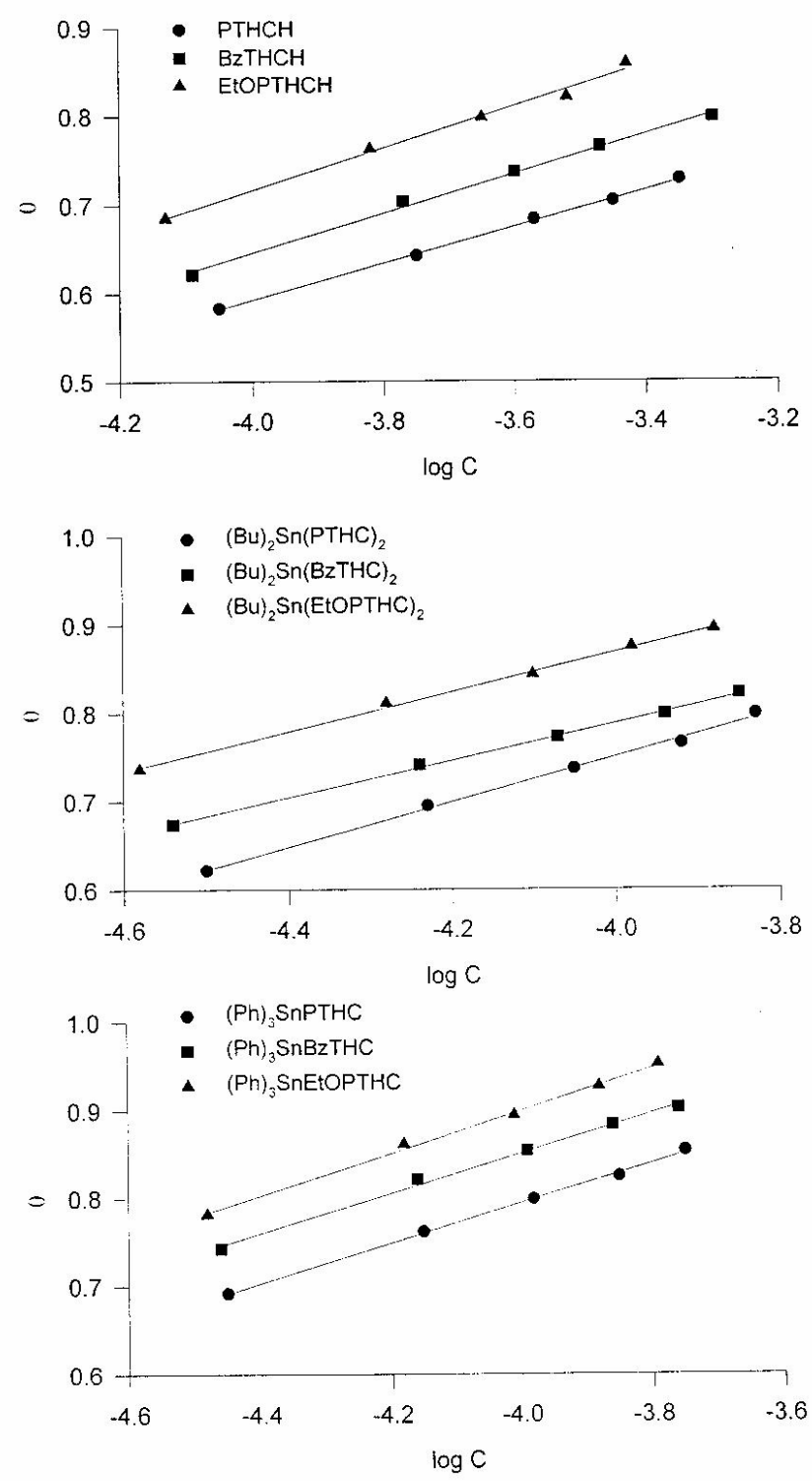

Figure 12. Temkin plots for dithiohydrazodicarbonamides and their dibutyltin (IV) and triphenyltin (IV) complexes.

\section{Conclusions}

1. All the studied compounds exhibited excellent corrosion inhibiting properties.

2. Among the ligands dithiohydrazodicarbonamides the best performance was shown by ethoxyphenyl derivative followed by benzyl and phenyl ones.

3. Complexation of the ligands in general resulted in appreciable increase in corrosion inhibition efficiency.

4. Triphenyltin complexes were found to be more effective than their dibutyltin analogues. 
5. Organotin complexes behaved predominantly as cathodic inhibitors.

\section{Acknowledgements}

Financial assistance from AICTE to R.B. Rastogi is gratefully acknowledge.

\section{References}

1. M.M. Singh, R.B. Rastogi, B.N. Upadhyay, M. Yadav, Mater. Chem. Phys. 80 (2003) 283-292.

2. M.M. Singh, R.B. Rastogi, B.N. Upadhyay, M. Yadav, Indian J. Chem. Technol. 10 (2003) 414-419.

3. R.B. Rastogi, M.M. Singh, M. Yadav, A. Bhattacharya, Wear 252 (2002) 686-692.

4. R.B. Rastogi, M.M. Singh, M. Yadav, Bull. Electrochem. In press.

5. R.N. Singh, V.B. Singh, Corros. Sci. 49 (1993) 569.

6. R.N. Singh, V.B. Singh, Mater. Trans. Japanese Inst. Metals. 38 (1997) 4853.

7. S.A.M.Y. Seliman, E.H. Ibrahim, J. Chem. Technol. Biotechnol. 46 (1989) 27.

8. S.A.M.Y. Seliman, E.H. Ibrahim, J. Chin. Chem. Soc. 37 (1990) 49.

9. M.M. Singh, R.B. Rastogi, B.N. Upadhyay, Corrosion 50 (1994) 620-625.

10. M.M. Singh, R.B. Rastogi, B.N. Upadhyay, Bull. Electrochem. 12 (1996) 26-30.

11. M.M. Singh, R.B. Rastogi, B.N. Upadhyay, M. Yadav, Indian J. Chem. Technol. 6 (1993) 93-99.

12. R.B. Rastogi, M.M. Singh, M. Yadav, K. Singh, Indian J. Eng. Mater. Sci. 10 (2003) 155-160.

13. J.A. Olabe, A.J. Arvia, Electrochim. Acta 14 (1969) 785.

14. U.R. Evans, "Oxidation and Corrosion of Metals", 3rd Ed., Arnold, London, 1968.

15. E. Gileadi, "Electrosorption", Plenum Press, New York, 1967 